\title{
Encefalopatía posterior reversible atípica secundaria a eclampsia. Presentación de un caso
}

\author{
Atypical reversible posterior encephalopathy due to eclampsia. Case presentation
}

\author{
Ana K. San Martín-Matamoros ${ }^{1}$, Joaquín Soto-Salamanca², José A. Cerón-Morales ${ }^{3}$, \\ Arturo García-Villaseñor y Michel D. Macías-Amezcua ${ }^{4 *}$ \\ ${ }^{1}$ Servicio de Ginecología y Obstetricia, Hospital de la Mujer, Puebla; ${ }^{2}$ Unidad de Cuidados Intensivos Adultos, Hospital de la Mujer, Puebla; ${ }^{3} S e r v i c i o$ \\ de Neurología, Hospital de la Mujer, Secretaría de Salud, Puebla; ${ }^{4}$ Genética Instituto ARTHIL, Puebla; ${ }^{5}$ Medicina Materno Fetal, Centro Medico \\ Nacional 20 de Noviembre, Instituto de Seguridad Social al Servicio de los Trabajadores del Estado, Ciudad de México. México
}

\section{Resumen}

Antecedentes: La encefalopatía posterior reversible se relaciona con enfermedades hipertensivas del embarazo, con clínica inespecífica. El diagnóstico se realiza mediante resonancia magnética y electroencefalograma, y el tratamiento oportuno evita complicaciones. Caso clínico: Primigesta de 15 años con embarazo pretérmino, hipertensión arterial y convulsiones tónico-clónicas, que desarrolló encefalopatía posterior atípica con múltiples lesiones cerebrales. Se administraron antihipertensivos, sin mejoría de los síntomas neurológicos. El manejo de esta patología depende de la situación desencadenante, no existe evidencia suficiente del soporte con medidas antiedema preventivas en pacientes con factores de riesgo. El retraso en el tratamiento oportuno y la presentación atípica favorecen la progresión de las secuelas neurológicas.

Palabras clave: Complicación del embarazo. Eclampsia. Encefalopatía hipertensiva.

\begin{abstract}
Background: Reversible posterior encephalopathy is related to hypertensive diseases of pregnancy, with a nonspecific clinic. The diagnosis is made by magnetic resonance and electroencephalogram, appropriate treatment prevents complications. Case report: 15-year-old primigesta with preterm pregnancy, arterial hypertension and tonic-clonic seizures, presents atypical posterior encephalopathy with multiple brain lesions, antihypertensives were administered without improvement of neurological symptoms. The management of this pathology depends on the triggering situation, there is insufficient evidence to support preventive anti-edema measures in patients with risk factors. The delay in timely treatment and atypical presentation favors the progression of neurological sequelae.
\end{abstract}

Key words: Pregnancy complications. Eclampsia. Hypertensive encephalopathy.

\footnotetext{
Correspondencia:

*Michel D. Macías-Amezcua

Avda. Félix Cuevas, 540

Col. Del Valle

Fecha de recepción: 27-09-2019

C.P. 03104, Ciudad de México, México

E-mail: mikedassaejv@gmail.com

Cir Cir. 2020;88(S1):87-90

Contents available at PubMed www.cirugiaycirujanos.com

0009-7411/@ 2020 Academia Mexicana de Cirugía. Publicado por Permanyer. Este es un artículo open access bajo la licencia CC BY-NC-ND (http://creativecommons.org/licenses/by-nc-nd/4.0/).
} 


\section{Introducción}

La encefalopatía posterior reversible fue descrita en 1996 por Hinchey y se caracteriza por edema cerebral vasogénico. En obstetricia, se asocia a enfermedades hipertensivas y se manifiesta con letargia, confusión, estupor, náuseas, convulsiones tónico-clónicas, escotomas, hemianopsia y ceguera cortical'.

La explicación fisiopatológica tiene dos hipótesis: una de ellas postula que la vasoconstricción arterial genera isquemia y edema citotóxico, y la otra considera que la hipertensión arterial sobrepasa los límites de la autorregulación cerebral, lo que conduce a vasodilatación, disfunción endotelial y edema vasogénico. La segunda es la hipótesis más aceptada al establecer una relación por la que en la mayoría de los casos el lóbulo occipital es el principal afectado debido a la menor inervación simpática de la circulación posterior ${ }^{1,2}$.

Se ha descrito su asociación con el uso de fármacos inmunosupresores y quimioterapéuticos. En la infancia, la principal etiología es la hipertensión arterial secundaria a enfermedad renal, glomerulonefritis 0 vasculitis, entre otras. Existen casos descritos en ausencia de hipertensión arterial. En las pacientes obstétricas es más frecuente en la eclampsia y en el síndrome de hemólisis, enzimas hepáticas elevadas y trombocitopenia (HELLP). Las características propias del síndrome HELLP pueden aumentar la respuesta vascular y el riesgo de sangrado, y predisponer a un curso clínico más agresivo y una mayor morbimortalidad materna ${ }^{3-5}$.

Se presenta un caso atípico de encefalopatia posterior reversible asociada a eclampsia, con lesiones en múltiples lóbulos cerebrales, en una mujer de 15 años primigesta que evolucionó a secuelas neurológicas, con el objetivo de reconocer la patología en obstetricia y promover el diagnóstico y el tratamiento oportunos.

\section{Presentación del caso}

Mujer de 15 años, primigesta con embarazo de 31 semanas, que presenta cefalea, desorientación, somnolencia y crisis convulsivas tónico-clónicas en tres ocasiones. Acude a urgencias con una presión arterial de 150/100 mmHg, taquicardia, Glasgow 12, sin signos de irritación meníngea, sin actividad uterina. El ultrasonido obstétrico evidencia un feto único vivo de 31 semanas, peso estimado 1755 gramos, frecuencia cardiaca fetal 140 latidos por minuto. El análisis bioquímico reporta hemoglobina $11 \mathrm{mg} / \mathrm{dl}$, plaquetas
$323,000 / \mu \mathrm{l}$, alanina aminotransferasa $144 \mathrm{U} / \mathrm{l}$ y aspartato aminotransferasa $133 \mathrm{U} / \mathrm{I}$.

Con diagnóstico de eclampsia se realizó cesárea bajo anestesia general, obteniendo un varón de 1820 gramos, Apgar 6-8, Capurro 34.2 semanas. La paciente ingresa a terapia intensiva, sin tratamiento antihipertensivo, con sulfato de magnesio como neuroprotector y bajo sedación e intubación orotraqueal. Se decide extubación a su ingreso y se espera a la mejoría del estado neurológico posterior al cese de los efectos sedantes; sin embargo, se mantiene somnolienta, pupilas mióticas e hiporreflécticas, respuesta a estímulos dolorosos, bioquímicamente sin cambios importantes, por lo que se sospecha una causa neurológica. Presenta posterior deterioro de la función ventilatoria que amerita soporte mecánico.

La tomografía computada simple de cráneo evidencia múltiples lesiones hipodensas de predominio corticosubcortical derecho, frontotemporal y parietal izquierdos, edema cerebral generalizado, sin desvío de la línea media, concluyendo en encefalopatía posterior reversible o evento vascular cerebral isquémico frontotemporal derecho. Se inició tratamiento con metilprednisolona y la paciente presentó evolución tórpida con hemiparesia corporal izquierda, Babinsky positivo derecho y descontrol hipertensivo. La resonancia magnética de cráneo simple y contrastada demostró lesiones sugestivas de encefalopatía posterior reversible bilateral abarcando ambos hemisferios, lóbulo frontal, parietal y occipital, confirmando el diagnóstico (Figs. 1 y 2). Como complemento diagnóstico se realizó un electroencefalograma que demostró cambios abruptos de voltaje y ondas lentas theta (Fig. 3).

Se suspendió la sedación y la paciente permaneció despierta, indiferente al medio, Glasgow 9, con midriasis. Se continuó el tratamiento con benzodiacepinas, antihipertensivos y antiepilépticos. Evolucionó hacia Glasgow 10, pupilas isocóricas, hiporreflécticas, fuerza muscular disminuida, respuesta plantar indiferente, estabilidad hemodinámica, control de la presión arterial, dependiente de traqueostomía con ventilación espontánea y gastrostomía funcional. El control bioquímico no mostró modificaciones respecto al inicial. Se decidió el egreso hospitalario con secuelas neurológicas irreversibles, indicando medidas de soporte y rehabilitación, con mal pronóstico para la vida y la función.

\section{Discusión}

El diagnóstico de encefalopatía posterior reversible fue descrito como un síndrome clínico caracterizado 


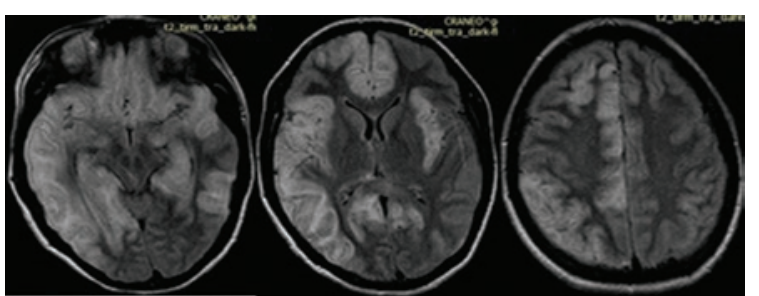

Figura 1. Resonancia magnética en plano axial, secuencia FLAIR T2. Se observan lesiones hiperintensas en ambos hemisferios cerebrales, en los lóbulos frontales, temporales y parietales, de forma asimétrica con predominio hacia el lado derecho, las cuales afectan la sustancia gris y la sustancia blanca, produciendo tumefacción del tejido cerebral.

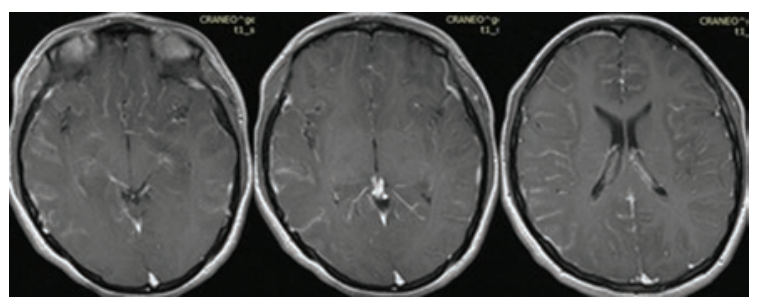

Figura 2. Resonancia magnética en secuencia $T 1$ con gadolinio en plano axial. Se observa discreto realce de contraste en las regiones temporales de predominio derecho, dirigido hacia los surcos. Imágenes sugestivas de síndrome de encefalopatía posterior reversible atípico.

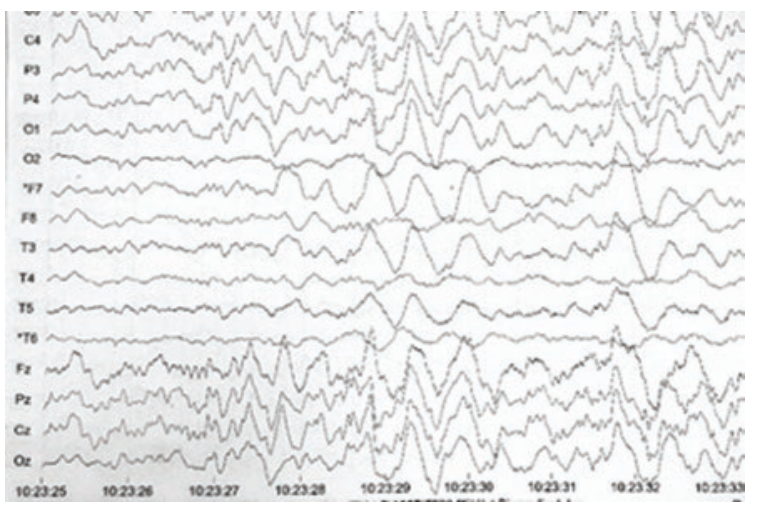

Figura 3. Electroencefalograma. Alteración de tipo lentificación paroxística por la presencia de cambios abruptos de voltaje con diferencia significativa entre las zonas anteriores y posteriores, así como brotes de ondas lentas theta de alto voltaje sincronizadas sobre las regiones frontocentrales de manera bilateral; asociado a cambios de la actividad cerebral de origen caudal de tallo cerebral.

por cefalea, alteración de la función mental, convulsiones y ceguera, relacionándose con hallazgos característicos en los estudios de imagen'. Se ha vinculado a enfermedad renal, infecciones agudas e hipertensión arterial. Fisiopatológicamente se explica por el desarrollo de edema vasogénico como consecuencia de un desequilibrio en la regulación vasomotora cerebral. En las pacientes obstétricas, en el $80 \%$ de los casos es consecuencia de hipertensión arterial, principalmente preeclampsia o eclampsia; sin embargo, hasta en el $20 \%$ se han observado lesiones características en ausencia de hipertensión arterial ${ }^{6,7}$.

Los síntomas descritos no son exclusivos de esta enfermedad, que con frecuencia no presenta un cuadro clínico florido, con crisis convulsivas en un 50$96 \%$ de las ocasiones ${ }^{3,6}$, encontrando mayor relación con manifestaciones aún más inespecíficas como cefalea y alteraciones visuales, siendo la asociación de estas dos últimas lo que habitualmente corresponde a encefalopatía posterior reversible. Algunos estudios en pacientes pediátricos y adolescentes muestran una gama aún mayor de manifestaciones, desde vómito hasta coma, por lo que se debe tomar en cuenta la edad cronológica para una intencionada búsqueda de la causa desencadenante ${ }^{6}$.

La sospecha diagnóstica se corrobora mediante métodos no invasivos, como con la tomografía computada simple o contrastada, en la que se evidenciará edema cerebral bilateral predominante en los lóbulos parietales y occipitales ${ }^{6,7}$. Dichas lesiones pueden orientar o bien servir como método para descartar otras patologías que pudieran ocasionar un cuadro clínico similar, como accidentes cerebrovasculares, síndrome de vasoconstricción cerebral, disección de la arteria cerebral, migraña, neuroinfecciones, enfermedades autoinmunitarias o metabólicas, y trastornos desmielinizantes ${ }^{8,9}$. En la mayoría de los casos, como en nuestra paciente, es el método de imagen inicial por ser útil para la exclusión de diagnósticos paralelos y establecer la pauta para la confirmación mediante otras técnicas más sensibles. En esta ocasión orientó hacia encefalopatía posterior o evento isquémico cerebral.

El siguiente paso fue la realización de resonancia magnética, método diagnóstico de elección en el que se pueden observar los signos patognomónicos: áreas hiperintensas en FLAIR y T2, ventanas en las que es posible visualizar lesiones con aumento del contenido de líquido, eliminando a su vez la señal proveniente del líquido cefalorraquíde $0^{3}$. Se utilizará la difusión para diferenciar entre encefalopatía posterior e isquemia cerebral. En la mayoría de los casos se observan lesiones en los lóbulos posteriores cerebrales, razón por la cual se le ha dado el nombre de encefalopatía posterior; sin embargo, existen algunos reportes de enfermedad atípica en los que se ve afectada la sustancia blanca de otros sitios cerebrales, como el cuerpo calloso y el tallo cerebral, los lóbulos 
frontales, los ganglios basales y el cerebelo; estos son menos frecuentes y, como en nuestro caso, su existencia puede ser un factor distractor para un correcto diagnóstico, además de implicar un peor pronóstico ${ }^{3,10}$.

Como método complementario para el diagnóstico se realizó un electroencefalograma en el que se registraron ondas theta lentas, que constituyen el trazo característico de esta patología.

El tratamiento se basa en la corrección de la causa desencadenante. En las pacientes obstétricas, la patología principalmente relacionada es la hipertensión arterial, por lo que el manejo es a base de antihipertensivos como labetalol, hidralazina o nifedipino, preferentemente en administración intravenosa. Es de suma importancia la administración de sulfato de magnesio como medida preventiva de episodios convulsivos $^{3}$. No existe evidencia suficiente que recomiende el uso de medidas antiedema cerebral, tanto en pacientes con diagnóstico de encefalopatía posterior como en aquellas con factores de riesgo para su desarrollo; no obstante, se ha demostrado que la instauración oportuna de tratamiento es primordial, y en esta paciente el antecedente de mal control prenatal nos hace pensar en un posible descontrol hipertensivo desde semanas de gestación previas con omisión de las medidas necesarias ${ }^{11}$.

Hasta el $90 \%$ de los pacientes presentan recuperación completa en la semana posterior al tratamiento de la patología desencadenante ${ }^{12}$; sin embargo, si no se trata de forma oportuna se produce edema citotóxico que puede ocasionar secuelas neurológicas irreversibles, principalmente asociadas a isquemia. Los casos en que la reversibilidad no es satisfactoria se asocian a la gravedad de la causa desencadenante, los factores de riesgo asociados y la extensión de las lesiones encefálicas, siendo peor el pronóstico cuantos más lóbulos cerebrales se vean dañados. No obstante, en algunos casos reportados en pacientes pediátricos no existe asociación entre el número de lesiones y la gravedad del cuadro ${ }^{6}$.

\section{Conclusiones}

No todos los casos de crisis convulsivas en el embarazo son secundarios a eclampsia per se. La encefalopatía posterior es una complicación muchas veces subdiagnosticada y que, de no tratarse de forma oportuna, puede ocasionar secuelas irreversibles neurológicas e impactar de forma negativa en el pronóstico y la calidad de vida de la paciente.

\section{Conflicto de intereses}

Los autores declaran que no existe conflicto de intereses.

\section{Responsabilidades éticas}

Protección de personas y animales. Los autores declaran que no se han realizado experimentos en seres humanos o animales para esta investigación.

Confidencialidad de los datos. Los autores declaran que han seguido los protocolos de sus centros de trabajo sobre la publicación de datos de pacientes.

Derecho a la privacidad y consentimiento informado. Los autores han obtenido el consentimiento informado de los pacientes y/o sujetos referidos en el artículo. Este documento obra en poder del autor de correspondencia.

\section{Bibliografía}

1. Torres MU, Delgado LV, Giraldo N, Ureña P, Franco S, Hernández OH. Síndrome de encefalopatía posterior reversible: reporte de un caso fatal y análisis de factores predictores de mal pronóstico. Biomédica. 2017;37:13-9.

2. Matías-Guiu JA, García-Ptacek S, Ordás CM, Marcos-Dolado A, Porta-Etessam J. Síndrome de encefalopatía posterior reversible recurrente con respuesta a nimodipino. Neurología. 2012;27:378-80.

3. Álvarez-Pabón Y, Beltrán-Avendaño M, Di Lizio-Miele KG. Síndrome de encefalopatía posterior reversible, eclampsia y síndrome de HELLP. Rev Chil Obstet Ginecol. 2017;82:280-91.

4. Gamnio B, Rodríguez A, Pandolfo S, Giachetto G, Cerisola A, Kanopa V. Encefalopatía posterior reversible en el curso de una emergencia hipertensiva, serie de cuatro niños hospitalizados en el Centro Hospitalario Pereira Rossell. Arch Pediatr Urug. 2018;89:171-8.

5. Gokhale A, Kimona A, Kantor S, Prakash S, Manhas Y. Posterior reversible leukoencephalopathy syndrome (PRES) in intensive care unit case series. Indian J Crit Care Med. 2017;21:772-8.

6. Gungor S, Kilic B, Tabel Y, Selimoglu A, Ozgen U, Yilmaz S. Clinical and imaging findings in childhood posterior reversible encephalopathy syndrome. Iran J Child Neurol. 2018;12:16-25.

7. Shankar J, Banfield J. Posterior reversible encephalopathy syndrome: a review. Can Assoc Radiol J. 2017;68:147-53.

8. Abalo-Lojo JM, Baleato-González S, González F. Cortical blindness secondary to posterior reversible encephalopathy syndrome, recovered by successful blood pressure management. Arq Bras Oftalmol. 2017;80:324-6.

9. Neill TA. Reversible posterior leukoencephalopathy syndrome. UpToDate. 2018. Disponible en: https://www.uptodate.com/contents/reversible-posterior-leukoencephalopathy-syndrome.

10. Martínez SE, García-Contreras ML, Manzella DJ, Brugnolo MR, Raab LA, Dobarro M. Síndrome de leucoencefalopatía posterior reversible. Medicina (Buenos Aires). 2017;77:233.

11. Lio CF, Lee YH, Chan HY, Yu CC, Peng NJ, Chan HP. Posterior reversible encephalopathy syndrome in a postpartum hemorrhagic woman without hypertension: a case report. Medicine (Baltimore). 2017;96:e6690.

12. Sudulagunta SR, Sodalagunta MB, Kumbhat M, Settikere Nataraju A. Posterior reversible encephalopathy síndrome (PRES). Oxf Med Case Reports. 2017;2017:omx011. 